\title{
Tricobezoar gástrico em cateto (Pecari tajacu Linnaeus, 1758) criado em cativeiro no semiárido potiguar: Relato de caso
}

\author{
Gastric trichobezoar in a collared peccary (Pecari tajacu Limnaeus, 1758) raised in captivity in the \\ semi-arid region of Rio Grande do Norte: Case report \\ Tricobezoar gástrico en un pecarí de collar (Pecari tajacu Linnaeus, 1758) criado en cautiverio en \\ la región semiárida de Rio Grande do Norte: Reporte de caso
}

Recebido: 14/04/2021 | Revisado: 20/04/2021 | Aceito: 25/04/2021 | Publicado: 10/05/2021

Ana Caroline Freitas Caetano de Sousa

ORCID: https://orcid.org/0000-0001-5883-9704

Universidade Federal Rural do Semi-Árido, Brasil

E-mail: carolfreitas04@outlook.com

Igor Renno Guimarães Lopes

ORCID: https://orcid.org/0000-0003-1832-4647

Universidade Federal Rural do Semi-Árido, Brasil E-mail: igorrenno@gmail.com

João Augusto Rodrigues Alves Diniz

ORCID: https://orcid.org/0000-0001-5329-9059

Universidade Federal Rural do Semi-Árido, Brasil E-mail: joao-diniz15@hotmail.com

Gabriela Rebouças de Oliveira

ORCID: https://orcid.org/0000-0002-3365-3361

Universidade Federal Rural do Semi-Árido, Brasil

E-mail: gabriela.reboucas.09@gmail.com

Ana Indira Bezerra Barros Gadelha

ORCID: https://orcid.org/0000-0001-7541-1646

Universidade Federal Rural do Semi-Árido, Brasil

E-mail: anaindirabezerra@hotmail.com

Jael Soares Batista

ORCID: https://orcid.org/0000-0001-9663-7468 Universidade Federal Rural do Semi-Árido, Brasil

E-mail: Jaelsoares@hotmail.com

Moacir Franco de Oliveira

ORCID: https://orcid.org/0000-0002-6269-0823

Universidade Federal Rural do Semi-Árido, Brasi E-mail: moacir@ufersa.edu.br

\begin{abstract}
Resumo
Bezoares são estruturas sólidas encontradas no trato gastrointestinal, que são formadas por agregação de material não digerível resultado da ingestão repetida, podendo ter um núcleo de cabelo ou pelos. Causando sinais clínicos resultantes de processos obstrutivos, intussuscepção, enteropatias e perfurações, geralmente, seus sinais são inespecíficos e depende da localização e grau de obstrução. Desta forma, o presente trabalho tem como objetivo relatar a presença de um tricobezoar gástrico em um exemplar de cateto (Pecari tajacu) criado em cativeiro. O animal é proveniente do Centro de Multiplicação em Animais Silvestres (CEMAS) da Universidade Federal Rural do Semi-Árido (UFERSA), este animal fazia parte de um projeto, no qual deveria ser feito o acompanhamento gestacional, sendo necessário a realização de ultrassonografia, porém, com o exame não foram observadas estruturas compatíveis com prenhes, mas durante o exame físico foi palpado na região abdominal uma massa de consistência firme. Como medida de controle, o animal foi submetido a eutanásia e realizado exame necroscópico. Ao acessar a cavidade abdominal foi possível notar volume alterado no estômago e a presença de um corpo estranho, com forma circular, e com posterior incisão da câmara gástrica foi verificada a presença de uma estrutura oval, cobertas por matéria vegetal e com presença de pelo em seu núcleo, com coloração que variava do verde a tonalidades de preto. Por meio destes achados e junto ao levantamento bibliográfico, conclui-se que se trata de um tricobezoar gástrico.
\end{abstract}

Palavras-chave: Animais silvestres; Clínica; Necroscopia.

\section{Abstract}

Bezoars are solid structures found in the gastrointestinal tract, they are formed by aggregation of non-digestible material resulting from repeated ingestion, may having a nucleus hair or fur. Causing signs presented of obstructive processes, 
intussusception, enteropathies and perforations, generally, their signs are nonspecific and depends on the location and degree of obstruction. Thus, the present work aims to report the presence of a gastric trichobezoar in a specimen of collared peccary (Pecari tajacu) raised in captivity. The animal comes from the Center for Multiplication in Wild Animals (CEMAS) of the Federal Rural University of the Semi-Arid (UFERSA) and make part of a project, in this project should be done the gestational monitoring, being necessary the accomplishment of ultrasound, however, with the examination, no structures compatible with the principles were observed, but during the physical examination, a mass of firm consistency was felt in the abdominal region. As a control measure, the animal was euthanized and performed a necroscopic examination. Upon accessing the abdominal cavity, it was possible to notice the altered volume in the stomach and the presence of a foreign body, with a circular shape, and with a subsequent incision of the gastric chamber, the presence of an oval structure, covered with material vegetable and with the presence of fur in the nucleus, this nucleus had a color ranging from green to shades of black. Through these findings and together with the bibliographic survey, it is concluded that it is a gastric trichobezoar.

Keywords: Wild animals; Clinic; Necroscopy.

\section{Resumen}

Los bezoares son estructuras sólidas que se encuentran en el tracto gastrointestinal, que se forman por agregación de material no digerible resultante de la ingestión repetida, y pueden tener un núcleo de pelo o cabello. Provocando signos clínicos derivados de procesos obstructivos, intususcepción, enteropatías y perforaciones, generalmente sus signos son inespecíficos y dependen de la ubicación y grado de obstrucción. Así, el presente trabajo tiene como objetivo reportar la presencia de un tricobezoar gástrico en un ejemplar de pecarí de collar (Pecari tajacu) criado en cautiverio. El animal proviene del Centro de Multiplicación en Animales Silvestres (CEMAS) de la Universidad Federal Rural del Semiárido (UFERSA). Sin embargo, con el examen no se observaron estructuras compatibles con gestantes, pero durante el examen físico, se sintió una masa de consistencia firme en la región abdominal. Como medida de control, se sacrificó al animal y se le realizó un examen necroscópico. Al acceder a la cavidad abdominal se pudo notar una alteración del volumen en el estómago y la presencia de un cuerpo extraño, de forma circular, y con una posterior incisión de la cámara gástrica, la presencia de una estructura ovalada, recubierta de planta. material y con la presencia de pelo en él, se verificó su núcleo, con un color que variaba desde el verde hasta las tonalidades del negro. A través de estos hallazgos y junto con el relevamiento bibliográfico, se concluye que se trata de un tricobezoar gástrico.

Palabras clave: Animales salvajes; Clínica; Necroscopia.

\section{Introdução}

Bezoares são estruturas sólidas compostas por substâncias estranhas, de origem orgânica ou inorgânica, encontradas no trato digestivo. Esses corpos estranhos geralmente são resultado da ingestão repetida de material que não é digerido pelo o trato digestório causando acúmulos no intestino ou no estômago. Sua composição pode ser variada e quando formada por pêlos é denominada tricobezoar (Mir, 2011; Machado et al., 2019). Os tricobezoares podem causar sinais clínicos resultantes de processos obstrutivos, intussuscepção, enteropatias e perfurações. Geralmente, apresentam-se com sinais inespecíficos e sua intensidade depende da localização e grau da obstrução (Wolfson, et al., 1987; Hobday, 2014).

O cateto (Pecari tajacu) é um mamífero silvestre pertencente à família Tayassuidae, possui estômago parcialmente compartimentado, podendo ser dividido em quatro câmaras gástricas (Toschi, 1974). Este encontra-se distribuído desde o sul dos Estados Unidos até o norte da Argentina (Garcia et al., 2009), e no Brasil, habita os diversos biomas, como a Mata Atlântica, Cerrado, Amazônia, Caatinga e Pantanal (Desbiez et al., 2012). É uma espécie que apresenta importância zootécnica devido a carne e couro de boa qualidade (Santos et al., 2009), atraindo, assim, sua criação em cativeiro (Pereira-Júnior et al., 2016), o que torna necessário a compreensão sobre patologias que acometem a espécie.

Desta forma, o presente trabalho tem como objetivo descrever a presença de tricobezoar gástrico em um exemplar de cateto (Pecari tajacu), criado em cativeiro no semiárido potiguar.

\section{Metodologia}

Este trabalho, é um relato de caso de cunho descritivo e de natureza qualitativa, sendo a coleta de dados através do acesso aos registros médicos e aos exames fornecidos, tendo o pesquisador a função de instrumento primordial (Pereira et al., 2018). No presente trabalho, foi realizada a coleta de dados registrados no exame necroscópico e histórico do animal. Sendo feito um 
levantamento bibliográfico, em bancos de dados nacionais e internacionais.

\section{Relato}

O animal deste estudo foi proveniente do Centro de Multiplicação de Animais Silvestres (CEMAS), da Universidade Federal Rural do Semi-Árido, Mossoró-RN, registrada no IBAMA (Instituto Brasileiro de Manejo e Recursos Naturais Renováveis), sob o número 14.789.12. Trata-se de uma fêmea que vivia em um recinto (1,5x20m) e encontrava-se com outra fêmea. Sua alimentação era à base de concentrado de milho, soja e farelo de trigo, frutas cítricas e vegetais verdes, com fornecimento de água ad libitum.

Os animais estavam em um recinto separado, pois faziam parte de um grupo de animais, nos quais era realizado o acompanhamento da gestação através do uso de ultrassonografia. A contenção foi realizada com auxílio de um puçá e posteriormente sedada com a aplicação intravenosa de propofol $(7 \mathrm{mg} / \mathrm{kg}-1)$. Durante a realização do exame físico foi possível identificar a partir da palpação abdominal uma estrutura firme na região de flanco esquerdo, no qual foi considerado como um corpo estranho. Como protocolo, era necessário que os animais passassem por um exame ultrassonográfico transretal, para a visualização de uma possível gestação, entretanto, não foi visualizada nenhuma estrutura compatível com prenhes.

Assim como medida de controle do criatório, o animal foi submetido a eutanásia e posteriormente foi realizada a necropsia. Tal medida serve como controle sanitário a fim de evitar que doenças se espalhem pelo plantel, visto que durante o exame ultrassonográfico não foi possível identificar a natureza do material firme na cavidade abdominal. Para eutanásia foi instituído protocolo anestésico com a medicação pré-anestésica, por via intramuscular, de acepromazina $(0,2 \mathrm{mg} / \mathrm{kg}-1)$ e associação de cetamina ( $15 \mathrm{mg} / \mathrm{kg}-1)$ e maleato de midazolam (3mg/kg-1) pela mesma via. Atingindo o plano anestésico desejado, foi utilizado cloreto de potássio (2,56 mEq.Kg-1) por via intravenosa. Constatado o óbito do animal, seguiu-se para a necropsia.

Durante o exame necroscópico, logo após a abertura da cavidade abdominal, foi possível notar volume alterado no estômago e a presença de um corpo estranho, com forma circular (Figura 1.A). Com a observação do local onde o corpo estranho estava, foi realizado um corte em uma das câmaras do estômago, no sentido longitudinal, para a exposição do lúmen estomacal, no qual notou-se a presença de uma massa sólida de com tonalidades verde enegrecidas (Figura 1.B e C), com conteúdo de odor fétido. 
Figura 1. Tricobezoar gástrico. A. temos presença do corpo estranho (Tricobezoar) em uma das câmaras digestivas no estômago do cateto $(P$. tajacu $)$ coberta por conteúdo gástrico. B e C. Visualização do tricobezoar observando um núcleo composto por pelo, e envolto por material vegetal. $[$ Barra $=1 \mathrm{~cm}]$.

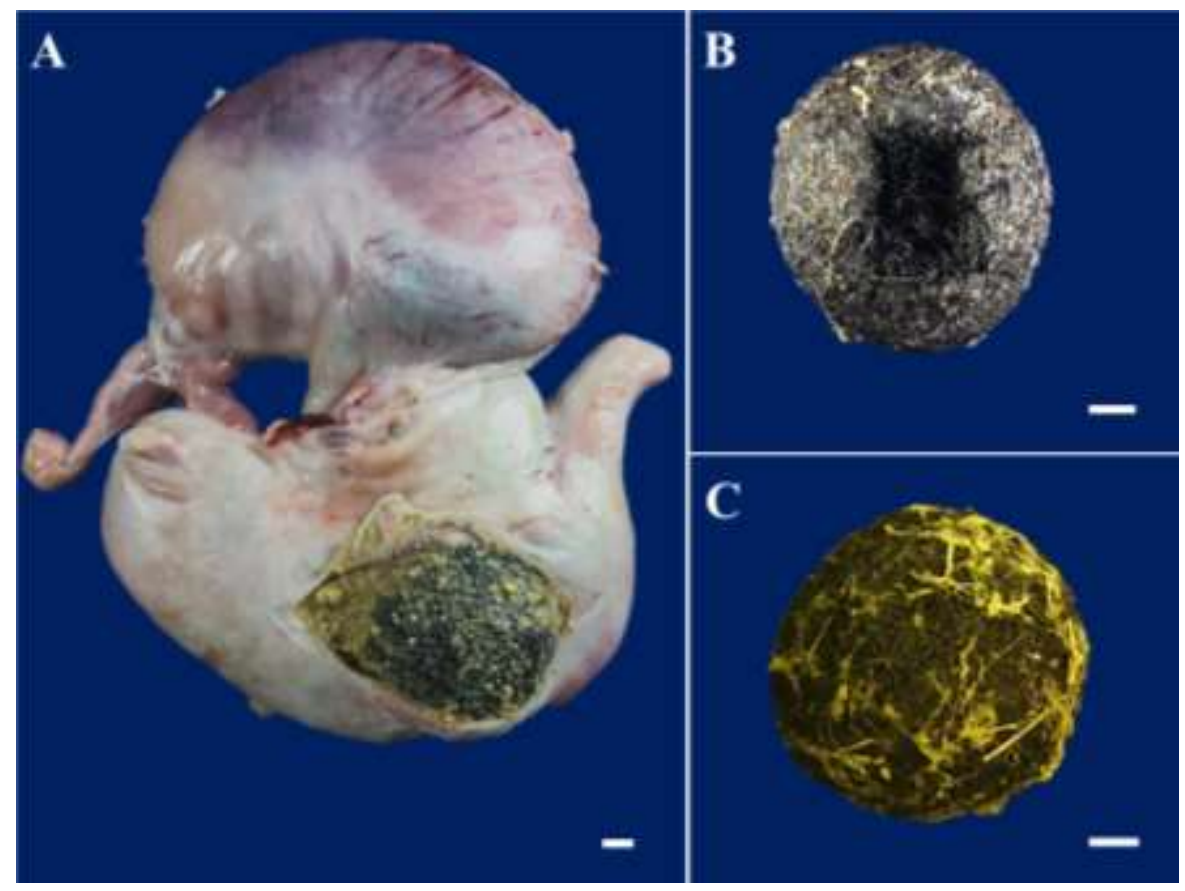

Fonte: Arquivo Pessoal.

O corpo estranho foi retirado e ao ser analisado notava-se uma massa densa entrelaçada de pelos e material não digerido, o que levou a confirmação de tricobezoar (Figura 1), e este foi submetido à morfometria e pesagem (Tabela 1), com o auxílio de paquímetro eletrônico (MITUTOYO®) e balança analítica de precisão (MARTE®).

Tabela 1. Dados da morfometria do tricobezoar. *mm-(milímetro) e g-(gramas).

\begin{tabular}{cccc}
\hline Comprimento & Largura & Espessura & Peso \\
$63,06 \mathrm{~mm}$ & $54,54 \mathrm{~mm}$ & $52,36 \mathrm{~mm}$ & $116.80 \mathrm{~g}$ \\
\hline
\end{tabular}

Fonte: Arquivo pessoal.

\section{Discussão}

Os bezoares são estruturas rígidas, de formato cúbico ou oval que são formadas no trato gastrointestinal por agregação de material não digerido, geralmente, de compostos alimentares ou pelos (Júnior et al., 2017). Dependendo da composição, este pode ser classificado em fitobezoar (matéria vegetal), tricobezoar (cabelo ou pelo), lactobezoar (fórmulas lácteas concentradas), farmacobezoar (fármacos) e bezoar alimentar (fórmulas entéricas enriquecidas) (Elzurumlu et al., 2005, Albernaz et al., 2017). O corpo estranho encontrado neste relato corresponde a um tricobezoar, obedecendo a classificação, pois seu núcleo era formado por pelo, envolto por material não digerido.

De acordo com Batista et al. (2014), quando certos tipos de corpos estranhos como, os enterólitos, apresentam-se como estruturas de pequenas proporções, estes podem ser eliminados facilmente pelas fezes, porém, quando apresentam grandes dimensões, podem ficar retidos em um desvio natural, ao longo do intestino, ou em alguma porção mais estreita do lúmen, o que podem levar a causar obstruções, vólvulos e intussuscepção. Nesses casos, ainda podem ocorrer necrose isquêmica, timpanismo secundário, além de morte por toxemia ou choque séptico. 
Desta forma, a utilização de técnicas de necrópsia é uma prática fundamental para a correção ou confirmação de um diagnóstico, infelizmente esta prática é bastante negligenciada por veterinários (Peixoto \& Barros, 1998). Neste caso, o exame necroscópico foi fundamental para o diagnóstico, e segundo Batista et al. (2014), o tricobezoar é um achado necroscópico comum em catetos em cativeiro, porém, neste estudo não foi elucidado a causa dessa patologia. Sabe-se que não existe predileção sexual, mas pode estar relacionado com dietas com baixas concentrações de fibras ou com excesso de auto-limpeza por conta do estresse (Martínez et al., 2018).

Aslanabi et al. (2020) indicam a ultrassonografia ou tomografia para auxiliar no diagnóstico de bezoares, sendo a endoscopia a melhor técnica a fornecer diagnóstico definitivo. Neste caso a ultrassonografia transretal apontou a presença de conteúdo abdominal, porém o diagnóstico definitivo foi somente via exame necroscópico, indo de acordo com o já relatado por Batista et al. (2014). Por se tratar de uma espécie silvestre e de difícil contenção, o uso da técnica ultrassonográfica por meio transretal não seja indicada para o diagnóstico dessa patologia, visto que o tipo de probe utilizada não alcançaria tal órgão devido a quantidade de estruturas entre eles, o que prejudicaria a visão do mesmo.

O diagnóstico precoce e a correção de manejo são fundamentais para um prognóstico favorável, evitando assim intervenções cirúrgicas e acometimentos clínicos (Aslanabadi et al., 2020). Por muitas vezes apresentar de forma subclínica, assim como foi observado neste caso, o tricobezoar pode se tornar problema grave a longo prazo podendo levar à ruptura (Muhammad et al., 2011) e formação de úlceras (Wilson et al., 2020). Embora, neste relato não tenham sido observadas alterações em outros órgãos, o odor fétido sentido na necropsia pode estar relacionado a fermentação de conteúdo alimentar.

A causa desta enfermidade pode ser por distúrbios psicológicos (Aslanabadi et al., 2020) ou alimentação desbalanceada (Martínez et al., 2018). Neste caso, ao conversar com o tratador para obter informações a fim de chegar a uma possível causa, foi informado que os animais apresentavam queda de pelo e este se acumulava no comedouro, sendo sugestivo de ingestão durante a alimentação, o que levou a formação do bezoar. Além disso, o comportamento de higienização pode ter contribuído para uma maior queda de pelos, e aumentando a ingestão e compactação no estômago do animal.

Em animais de produção, o tratamento de bezoares pode ser feito com o uso de enzimas para dissolver o bezoar, podendo ser usado, parafina líquida, papaína (mamão) e bromelina (abacaxi) (Nowland, 2015, Júnior et al., 2017). Em alguns casos já foram relatados o uso de substâncias oleosas, porém, não tiveram um resultado satisfatório sendo indicado a intervenção cirúrgica (Júnior et al., 2017). Neste caso, por se tratar de um tricobezoar, com um volume considerável o uso de óleo também não seria satisfatório.

\section{Conclusão}

De acordo com os achados, a estrutura firme e circular trata-se de um tricobezoar gástrico e sua formação não foi devido a distúrbios psicológicos ou alimentares, sendo a provável causa pela ingestão ocasional de pelos que se misturavam à ração dentro do comedouro.

Entretanto mais estudos devem ser elaborados tanto na parte da clínica médica veterinária de animais silvestres para auxiliar em diagnósticos precoces e assim planejar um tratamento que possa prolongar a vida do animal para que não seja necessário à sua eutanásia. Como também mais estudos que abordam o manejo nutricional para animais que vivem ex situ e em boas práticas de manejo dentro dos recintos evitando assim que os animais façam a ingestão de materiais estranhos a sua alimentação.

\section{Agradecimentos}

Gostaríamos de agradecer ao Centro de Multiplicação de Animais Silvestres (CEMAS), da Universidade Federal Rural do Semi-Árido pelo o fornecimento do material do estudo, bem como, também gostaríamos de agradecer ao Conselho Nacional 
de Desenvolvimento Científico e Tecnológico (CNPq) e à Coordenação de Aperfeiçoamento de Pessoal de Nível Superior Brasil (CAPES, 001).

\section{Referências}

Albernaz, V. G. P., Conceição, R. T., Eising, T. C., de Almeida Fabris, I., Mamprim, M. J., \& Rahal, S. C. (2017). Partial Obstruction of the Small Intestine by a Trichobezoar in a Dog. Acta Scientiae Veterinariae, $45,5$.

Aslanabadi, S., Badebarin, D., Parsay, S., Jamshidi, M., Farhadi, E., e Ladan, A. (2020). Um caso de trichobezoar gástrico gigante. Revista Iraniana de Cirurgia Pediátrica, 5 (2), 98-101.

Batista, J. S., Olinda, R. G., Rodrigues, C. M., Silva, T. M., Vale, R. G., Viana, G. A., Oliveira, A. F., Oliveira, M. F. (2014). Postmortem findings in collared peccaries raised in captivity in northeastern Brazil. Pesquisa Veterinária Brasileira, 34 (11), 1101-1108.

Desbiez, A. L. J., Keuroghlian, A., de Mello Beisiegel, B., Medici, E. P., Gatti, A., Pontes, A. R. M., Campos, C. B., Tófoli, C. F., Junior, E. A. M., Azevedo, F. C., Pinho, G. M., Cordeiro, J. L. P., Júnior, T. S. S., Morais, A. A. M., Mangini, P. R., Flesher, K., Rodrigues, F. Almeida, L. B., \& de Almeida, L. B. (2012). Avaliação do risco de extinção do cateto Pecari tajacu Linnaeus, 1758, no Brasil. Biodiversidade Brasileira-BioBrasil, (1), 74-83.

Erzurumlu, K; Malazgirt, Z.; Bektas, A.; Dervisoglu, A.; Polat, C.; Senyurek, G., Yetim, I., Ozkan, K. (2005). Gastrointestinal bezoars: a retrospective analysis of 34 cases. World journal of gastroenterology: WJG, 11 (12), 1813.

Garcia, A. R., Kahwage, P. R., \& Ohashi, O. M. (2009). Aspectos reprodutivos de caititus (Tayassu tajacu). Embrapa Amazônia Oriental-Artigo em periódico indexado (ALICE).

Hobday, M. M., Pachtinger, G. E., Drobatz, K. J., \& Syring, R. S. (2014). Linear versus non-linear gastrointestinal foreign bodies in 499 dogs: clinical presentation, management and short-term outcome. Journal of Small Animal Practice, 55 (11), 560-565.

Júnior, F. D. C. C., Silva, N. C. B., da Silva, Y. A., Pereira, A. M., de Sousa Mendonça, W., Junior, F. S. F., \& da Silva Tenório, T. G. (2017). Obstrução intestinal por fitobezoar em bovinos: Revisão. PUBVET, 11, 538-645.

Machado, R. S., Silva, R. S., Angst, J. P. S., Henrich, K., Tatsch, F. M., Rigão, G. C., Dutra, A. L., Rosa, L. D. (2019). Obstrução intestinal por tricobezoar em um cão: relato de caso. Brazilian Journal of Development, 5 (8), 12243-12249.

Martínez, M. Á. J., Gasper, D.J., Muciño, M. D. C. C. E., Terio, K. A. (2018). Suidae e Tayassuidae. In: Patologia de animais selvagens e animais do zoológico. Academic Press, 207-228.

MIR, A. Trichobezoar. (2011). Journal of the College of Physicians and Surgeons--pakistan: JCPSP, 21 (12), 763-765.

Muhammad, M., Banyigyi, S. A., Tafarki, E. A., Ighodalo, E. T., Benshak, J., Asala, O., Rimfa, A., Ibu, J. O \& Ehizobolo, D. (2011). Trichobezoar as cause of anorexia in a West African dwarf goat. Nigerian Veterinary Journal, 32(4).

Nowland, M. H., Brammer, D. W., Garcia, A., \& Rush, H. G. (2015). Biology and diseases of rabbits. In: Laboratory animal medicine, $3^{\circ}$. Academic Press, pp. 411-461.

Peixoto, P. V., \& Barros, C. S. (1998). A importância da necropsia em medicina veterinária. Pesquisa Veterinária Brasileira, 18 (3-4), $132-134$.

Pereira, A. S., Shitsuka, D. M., Parreira, F. J., \& Shitsuka, R. (2018). Metodologia da pesquisa científica. UFSM.

Pereira-Junior, R. A., Sousa, S. A., Oliveira, M. C., Valadares, A. D. A., Hoppe, E. G., \& Almeida, K. S. (2016). Fauna helmintológica de catetos (Tayassu tajacu Linnaeus, 1758) procedentes da Amazônia Brasileira1. Pesquisa Veterinária Brasileira, 36 (11), 1109-1115.

Santos, D. O., Mendes, A., da Cunha Nogueira, S. S., \& Nogueira Filho, S. L. G. (2009). Criação comercial de caititus (" Pecari tajacu"): uma alternativa para o agronegócio. Revista Brasileira de Saúde e Produção Animal, 10 (1).

Toschi, A. (1974). Tayassu tajacu. In: Enciclopedia Monográfica de Ciencias Naturales, Editorial Aguilar. 5:370-371.

Wilson, J. M., Wallace, C. K., Brice, A. K., Makaron L. (2020). "Tricobezoares mineralizados em um macaco Rhesus (Macaca mulatta). Journal of Medical Primatology, 49 (3), 158-161.

Wolfson, P. J., Fabius, R. J \& Leibowitz, A. N. (1987). The Rapunzel syndrome: an unusual trichobezoar. American Journal of Gastroenterology (Springer Nature), 82 (4). 